\title{
Erratum to: Multiple solutions to logarithmic Schrödinger equations with periodic potential
}

\author{
Marco Squassina $^{1} \cdot$ Andrzej Szulkin ${ }^{2}$
}

Published online: 13 April 2017

(C) Springer-Verlag Berlin Heidelberg 2017

\section{Erratum to: Calc. Var. (2015) 54:585-597 DOI 10.1007/s00526-014-0796-8}

In our paper [3], there are some technical errors which do not affect the validity of the results but require modification of the arguments. We are grateful to Kazunaga Tanaka, Chengxiang Zhang, and Qingye Zhang for kindly pointing out these errors.

We shall use the same notation as in [3]. Lemma 2.4 is not correct as stated and requires some changes. For $u \in D(J)$ and $z \in C_{0}^{\infty}\left(\mathbb{R}^{N}\right)$, let

$$
\left\langle J^{\prime}(u), z\right\rangle:=\left\langle\Phi^{\prime}(u), z\right\rangle+\int_{\mathbb{R}^{N}} Q(x) F_{1}^{\prime}(u) z d x .
$$

$J^{\prime}(u)$ is a densely defined linear operator, and we may define

$$
\left\|J^{\prime}(u)\right\|:=\sup \left\{\left\langle J^{\prime}(u), z\right\rangle: z \in C_{0}^{\infty}\left(\mathbb{R}^{N}\right),\|z\| \leq 1\right\} .
$$

If $\left\|J^{\prime}(u)\right\|$ is finite, then $J^{\prime}(u)$ may be extended to a bounded operator in $E$ and is thus an element of $E^{\prime}$.

Lemma 2.4 Let $u \in D(J)$ and $J^{\prime}(u) \in E^{\prime}$. Then $w \in \partial J(u)$, i.e.

$$
\left\langle\Phi^{\prime}(u), v-u\right\rangle+\Psi(v)-\Psi(u) \geq\langle w, v-u\rangle \text { for all } v \in E
$$

The online version of the original article can be found under doi:10.1007/s00526-014-0796-8.

Marco Squassina

marco.squassina@unicatt.it

Andrzej Szulkin

andrzejs@math.su.se

1 Dipartimento di Matematica e Fisica, Università Cattolica del Sacro Cuore, Via dei Musei 41, 25121 Brescia, Italy

2 Department of Mathematics, Stockholm University, 10691 Stockholm, Sweden 
if and only if $w=J^{\prime}(u)$. If $\left(u_{n}\right)$ is a Palais-Smale sequence, then $J^{\prime}\left(u_{n}\right) \in E^{\prime}$ and $J^{\prime}\left(u_{n}\right) \rightarrow$ 0 .

Proof Insert $v=u+t z$, where $t>0$ and $z \in C_{0}^{\infty}\left(\mathbb{R}^{N}\right)$, into (*). This gives

$$
\left\langle\Phi^{\prime}(u), z\right\rangle+\int_{\mathbb{R}^{N}} Q(x) \frac{F_{1}(u+t z)-F_{1}(u)}{t} d x \geq\langle w, z\rangle .
$$

Since the integrand is 0 for $x \notin \operatorname{supp} z$, we can pass to the limit as $t \rightarrow 0$, and we obtain

$$
\left\langle\Phi^{\prime}(u), z\right\rangle+\int_{\mathbb{R}^{N}} Q(x) F_{1}^{\prime}(u) z d x \geq\langle w, z\rangle
$$

[recall that by Lemma 2.2, $\Psi \in C^{1}\left(H^{1}(\Omega), \mathbb{R}\right)$ if $\Omega$ is bounded]. Since this also holds for $-z$

$$
\left\langle J^{\prime}(u), z\right\rangle=\left\langle\Phi^{\prime}(u), z\right\rangle+\int_{\mathbb{R}^{N}} Q(x) F_{1}^{\prime}(u) z d x=\langle w, z\rangle \text { for all } z \in C_{0}^{\infty}\left(\mathbb{R}^{N}\right) .
$$

By density of $C_{0}^{\infty}\left(\mathbb{R}^{N}\right)$ in $E, w$ is unique and $J^{\prime}(u)=w$.

If $\left(u_{n}\right)$ is a Palais-Smale sequence, then by definition

$$
\left\langle\Phi^{\prime}\left(u_{n}\right), v-u_{n}\right\rangle+\Psi(v)-\Psi\left(u_{n}\right) \geq-\varepsilon_{n}\left\|v-u_{n}\right\| \text { for all } v \in E,
$$

where $\varepsilon_{n} \rightarrow 0^{+}$. Letting $v=u_{n}+t z$, where $t>0$ and $z \in C_{0}^{\infty}\left(\mathbb{R}^{N}\right)$, we have

$$
\left\langle\Phi^{\prime}\left(u_{n}\right), z\right\rangle+\int_{\mathbb{R}^{N}} Q(x) \frac{F_{1}\left(u_{n}+t z\right)-F_{1}\left(u_{n}\right)}{t} d x \geq-\varepsilon_{n}\|z\|,
$$

so passing to the limit as $t \rightarrow 0$, we obtain

$$
\left\langle J^{\prime}\left(u_{n}\right), z\right\rangle \geq-\varepsilon_{n}\|z\|, \quad z \in C_{0}^{\infty}\left(\mathbb{R}^{N}\right) .
$$

Hence $J^{\prime}\left(u_{n}\right) \in E^{\prime}$ and $\left\|J^{\prime}\left(u_{n}\right)\right\| \leq \varepsilon_{n} \rightarrow 0$.

The proof of Lemma 2.7 in [3] contains a gap which may not be easy to remove. Instead we prove a different (and in fact simpler!) version of this lemma. We employ an idea from [1]. For $d>0$ and $u \in J^{d}$, let

$$
\mu_{d}(u):=\inf _{a \in J^{d}}\left\{\left\|J^{\prime}(a)\right\|+\|u-a\|\right\} .
$$

It is easy to see that $\mu_{d}(u)<\infty$. Since $\mu_{d}(u) \leq\left\|J^{\prime}(u)\right\|$, it is clear that $\mu_{d}\left(u_{n}\right) \rightarrow 0$ if $J^{\prime}\left(u_{n}\right) \rightarrow 0$. On the other hand, if $\mu_{d}\left(u_{n}\right) \rightarrow 0$, then $J^{\prime}\left(a_{n}\right) \rightarrow 0$ for some $\left(a_{n}\right)$ such that $u_{n}-a_{n} \rightarrow 0$. The mapping $\mu_{d}$ is Lipschitz continuous. Indeed, for $u, v, a \in J^{d}$,

$$
\mu_{d}(u) \leq\left\|J^{\prime}(a)\right\|+\|u-a\| \leq\left\|J^{\prime}(a)\right\|+\|v-a\|+\|u-v\|,
$$

so taking the infimum over $a$ on the right-hand side we obtain $\mu_{d}(u) \leq \mu_{d}(v)+\|u-v\|$, and similarly, $\mu_{d}(v) \leq \mu_{d}(u)+\|u-v\|$. Hence $\left|\mu_{d}(u)-\mu_{d}(v)\right| \leq\|u-v\|$.

Lemma 2.7 For each $d>0$ there exists a locally Lipschitz continuous vector field $H$ : $J^{d} \backslash K \rightarrow E$ with the following properties:

(i) $\|H(u)\| \leq 1$.

(ii) $\left\langle J^{\prime}(u), H(u)\right\rangle>\frac{1}{2} \mu_{d}(u)$.

(iii) $H$ has locally compact support, i.e., for each $u_{0} \in J^{d} \backslash K$, there exists a neighbourhood $U_{0}$ of $u_{0}$ in $J^{d} \backslash K$ and $R>0$ such that $u(x)=0$ for all $|x| \geq R$ and $u \in U_{0}$. 
(iv) $H$ is odd in $u$.

Proof Let $\tilde{u} \in J^{d} \backslash K$. As $\left\|J^{\prime}(\widetilde{u})\right\| \geq \mu_{d}(\widetilde{u})$, there exists $\widetilde{v} \in C_{0}^{\infty}\left(\mathbb{R}^{N}\right),\|\widetilde{v}\|=1$ such that $\left\langle J^{\prime}(\tilde{u}), \widetilde{v}\right\rangle>\frac{1}{2} \mu_{d}(\widetilde{u})$ (this also holds if $\left\|J^{\prime}(\tilde{u})\right\|=\infty$ ). Since $\widetilde{v}$ has compact support, $u \mapsto\left\langle J^{\prime}(u), \widetilde{v}\right\rangle$ is continuous according to Lemma 2.2, and since also $\mu_{d}$ is continuous, $\left\langle J^{\prime}(u), \widetilde{v}\right\rangle>\frac{1}{2} \mu_{d}(u)$ for all $u$ in a neighbourhood $W(\tilde{u})$ of $\tilde{u}$. Clearly, $(W(\tilde{u}))$ is a covering of $J^{d} \backslash K$. Since $E$ is metric and hence paracompact, we can find a locally finite refinement $\left(W_{j}\right)$ and points $v_{j} \in C_{0}^{\infty}\left(\mathbb{R}^{N}\right)$ such that $\left\langle J^{\prime}(u), v_{j}\right\rangle>\frac{1}{2} \mu_{d}(u)$ for $u \in W_{j}$. Let now

$$
H_{1}(u):=\sum_{j=1}^{\infty} \rho_{j}(u) v_{j} \quad \text { and } \quad H(u):=\frac{1}{2}\left(H_{1}(u)-H_{1}(-u)\right),
$$

where $\left(\rho_{j}\right)$ is a Lipschitz continuous partition of unity subordinate to the cover $\left(W_{j}\right)$ of $J^{d} \backslash K$. It is easy to see that (i)-(iv) hold.

The change in Lemma 2.7 prompts some (slight) changes in the proofs of Lemmas 2.13 and 2.14. First of all, the flow $\eta$ in (2.4) should be considered for $u \in J^{d} \backslash K$ instead of $u \in D(J) \backslash K$. Likewise, the flow $\tilde{\eta}$ on p. 595 should be considered for $u \in J^{d+2 \varepsilon_{0}}$. On p. 592, line $17,-z(\eta(t, u))$ should be replaced by $-\frac{1}{2} \mu_{d}(\eta(t, u))$ and similarly, at the end of p. 592 in the definition of $\kappa_{n}, z(\eta(s, u))$ should read $\frac{1}{2} \mu_{d}(\eta(s, u))$. Then $\kappa_{n} \rightarrow 0$ as before and by (ii) of Lemma 2.7 and the properties of $\mu_{d}$, there exist $s_{n}^{1} \in\left[t_{n}, t_{n}^{1}\right]$ and $u_{n}^{1}$ such that $u_{n}^{1}-\eta\left(s_{n}^{1}, u\right) \rightarrow 0, J^{\prime}\left(u_{n}^{1}\right) \rightarrow 0$ and $J\left(u_{n}^{1}\right) \leq d$. Similarly we get $s_{n}^{2} \in\left[t_{n}^{1}, t_{n+1}\right]$ and $u_{n}^{2}$ such that $u_{n}^{2}-\eta\left(s_{n}^{2}, u\right) \rightarrow 0, J^{\prime}\left(u_{n}^{2}\right) \rightarrow 0$ and $J\left(u_{n}^{2}\right) \leq d$. Now the proof continues as in [3].

A similar change must be made in the proof of Lemma 2.14. In the definition of $\tau$, $z(u)$ should be replaced by $\frac{1}{2} \mu_{d+2 \varepsilon_{0}}(u)$. If $\tau=0$, we find a sequence $w_{n}^{1} \in J_{d-2 \varepsilon_{0}}^{d+2 \varepsilon_{0}} \cap$ $U_{\delta}\left(K_{d}\right) \backslash U_{\delta / 2}\left(K_{d}\right)$ with $\mu_{d+2 \varepsilon_{0}}\left(w_{n}^{1}\right) \rightarrow 0$ and then $u_{n}^{1}$ such that $u_{n}^{1}-w_{n}^{1} \rightarrow 0, J^{\prime}\left(u_{n}^{1}\right) \rightarrow 0$ and $J\left(u_{n}^{1}\right) \leq d+2 \varepsilon_{0}$. The rest of the proof is the same as in [3].

At the end of the proof of Theorem 1.1 it is not clear that $T$ exists as claimed (the reason being the lack of continuity of $J$ ). On the other hand, $J$ is continuous on sets having compact support, see Lemma 2.2. In order to take advantage of this fact we need to slightly redefine the family $\mathcal{H}$ on p. 594. Let

$$
\mathcal{H}:=\{h: D(J) \rightarrow E, h \text { odd homeomorphism onto } h(D(J)), J(h(u)) \leq J(u)
$$

for all $u \in D(J)$ and if $A \in \Sigma$ has compact support, then so does $h(A)\}$.

Since for each $k \geq 1$ there exists a $k$-dimensional subspace $E_{k} \subset C_{0}^{\infty}\left(\mathbb{R}^{N}\right)$, according to Lemma 2.16 there are sets $A \in \Sigma$ having compact support and pseudoindex $i^{*}(A) \geq k$. Therefore, the numbers

$$
d_{k}:=\inf \left\{\sup _{u \in A} J(u): i^{*}(A) \geq k \text { and } A \text { have compact support }\right\}
$$

are well defined for all $k \geq 1$. Moreover, $d_{k} \geq b$ by Lemma 2.15 .

Let $\widetilde{\eta}$ (with $u \in J^{d+2 \varepsilon_{0}}$ ) be as in the proof of Theorem 1.1. We must show that there exists $T>0$ such that $J(\tilde{\eta}(T, u))<d-\varepsilon$ for all $u \in B:=A \backslash U$. It is clear by construction of the vector field $H$ that $\tilde{\eta}([0, t] \times B)$ has compact support for any $t>0$. Given $u_{0} \in B$, we have $J\left(\tilde{\eta}\left(T_{0}, u_{0}\right)\right)<d-\varepsilon$ for some $T_{0}>0$. Since the restriction of $J$ to $\tilde{\eta}\left(\left[0, T_{0}\right] \times B\right)$ is continuous according to Lemma 2.2, we can find a neighbourhood $V_{0}$ of $u_{0}$ in $B$ such that $J\left(\tilde{\eta}\left(T_{0}, u\right)\right)<d-\varepsilon$ for all $u \in V_{0}$. Now we use compactness in order to find a finite 
covering $V_{i}$ of $B$ and take $T:=\max _{i} T_{i}$, where $T_{i}$ is such that $J\left(\widetilde{\eta}\left(T_{i}, u\right)\right)<d-\varepsilon$ for all $u \in V_{i}$. In order to get $\tilde{\eta}(T, \cdot) \in \mathcal{H}$, we need $\tilde{\eta}$ to be defined for all $u \in D(J)$ (and not only for $u \in J^{d+2 \varepsilon_{0}}$ ). To achieve this, it suffices to put $H(u)=0$ if $u \notin \operatorname{supp} \rho_{j}$ for any $j$.

A similar change needs to be made in the proof of Theorem 1.2: $\Gamma$ should consist of paths $\alpha$ which have compact support. See also Remark 3.8 in [2].

\section{References}

1. de Paiva, F.O., Kryszewski, W., Szulkin, A.: Generalized Nehari manifold and semilinear Schrödinger equation with weak monotonicity condition on the nonlinear term, Proc. AMS (to appear). arXiv:1609.04611

2. Ji, C., Szulkin, A.: A logarithmic Schrödinger equation with asymptotic conditions on the potential. J. Math. Anal. Appl. 437, 241-254 (2016)

3. Squassina, M., Szulkin, A.: Multiple solutions to logarithmic Schrödinger equations with periodic potential. Calc. Var. Partial Differ. Equ. 54, 585-597 (2015) 